University of the Pacific

Scholarly Commons

6-19-2013

\title{
Influence of cochlear implantation on sentence intelligibility and duration
}

\section{Olga Peskova}

The University of Texas at Dallas

Nirmal Kumar Srinivasan

\section{Sujin Shin}

Madhu Sundarrajan

University of the Pacific, msundarrajan@pacific.edu

Emily Tobey

Follow this and additional works at: https://scholarlycommons.pacific.edu/shs-all

Part of the Medicine and Health Sciences Commons

\section{Recommended Citation}

Peskova, Olga; Srinivasan, Nirmal Kumar; Shin, Sujin; Sundarrajan, Madhu; and Tobey, Emily, "Influence of cochlear implantation on sentence intelligibility and duration" (2013). All Faculty Scholarship. 108.

https://scholarlycommons.pacific.edu/shs-all/108

This Article is brought to you for free and open access by the School of Health Sciences at Scholarly Commons. It has been accepted for inclusion in All Faculty Scholarship by an authorized administrator of Scholarly Commons. For more information, please contact mgibney@pacific.edu. 
Influence of cochlear implantation on sentence intelligibility and duration.

Olga Peskova, Nirmal Kumar Srinivasan, Sujin Shin, Madhu Sundarrajan, and Emily Tobey

Citation: Proc. Mtgs. Acoust. 19, 060131 (2013); doi: 10.1121/1.4800733

View online: https://doi.org/10.1121/1.4800733

View Table of Contents: https://asa.scitation.org/toc/pma/19/1

Published by the Acoustical Society of America

\section{ARTICLES YOU MAY BE INTERESTED IN}

Effects of auditory feedback on fricatives produced by cochlear-implanted adults and children: Acoustic and perceptual evidence

The Journal of the Acoustical Society of America 119, 1626 (2006); https://doi.org/10.1121/1.2167149 


\section{Proceedings of Meetings on Acoustics}
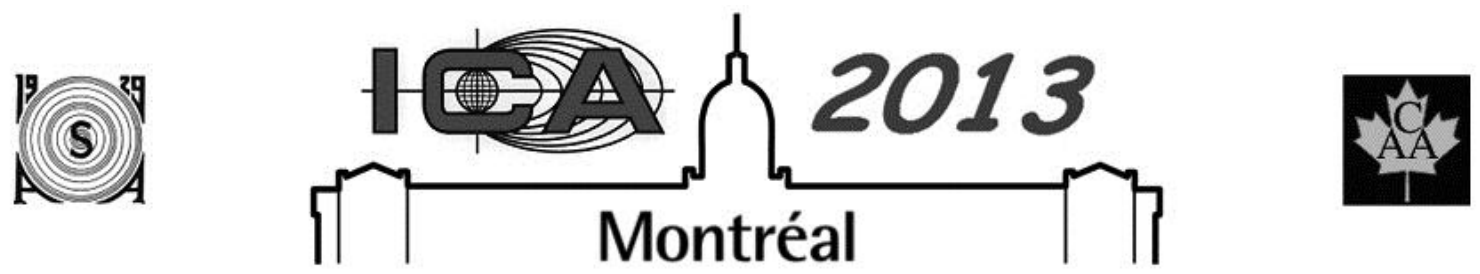

ICA 2013 Montreal

Montreal, Canada

2 - 7 June 2013

Speech Communication

Session 2pSCb: Speech Intelligibility (Poster Session)

\section{2pSCb23. Influence of cochlear implantation on sentence intelligibility and duration.}

Olga Peskova*, Nirmal Kumar Srinivasan, Sujin Shin, Madhu Sundarrajan and Emily Tobey

*Corresponding author's address: Dallas Cochlear Implant Program, The University of Texas at Dallas, Communication Sciences and Disorders, Richardson, TX 75080, oxp100020@utdallas.edu

Cochlear implants (CI) allow children with hearing loss (HL) to achieve speech perception and production outcomes that make their spoken speech understandable to normal hearing adult listeners. This capability is characterize by wide variability of scores. In order to understand the factors that contribute to the overall variability, we investigated the effects of duration of cochlear implantation on speech intelligibility and sentence duration over time. Participants were 107 children implanted between the ages of 2 and 4 and tested at 2 time points there they were 8 and 16 years old. Participants repeated McGarr sentences, which vary in length from 3 to 5 to 7 syllables. Recording were analyze using acoustic software to designate the beginning and end of each sentence in listeners who only heard one sentence from one child. Speech intelligibility scores were related statistically to the duration of each sentence. Durations of sentences that approximated that of normal hearing listeners were those with high intelligibility judgment. In addition, it appears that the children with the longest experience of CI use continue to improve their intelligibility. (Sponsored by NIH).

Published by the Acoustical Society of America through the American Institute of Physics 


\section{INTRODUCTION}

Speech intelligibility is a measure of the amount of speech understood by listeners. However, the speech intelligibility of people with hearing loss (HL) often is diminished, resulting in difficulties during daily human interactions with normal hearing individuals. Indeed, Gold (1980) reviewed a series of studies and indicated that "...only about $20 \%$ of the speech output of the deaf is understood by inexperienced listeners" (p. 397) (Gold, 1980). When new technologies such as cochlear implantation arrived in the 1990's, the hearing of individuals with HL improved and changed communication abilities dramatically, as reflected in improvements in speech intelligibility.

Researchers report that after cochlear implantation, children with HL improve their ability to hear, perceive and produce speech. These improvements lead to increased speech intelligibility and aid their overall adaptation to the society (Tobey et al., 1991; Robbins et al., 1995; Miyamoto et al., 1996; Vieu et al., 1998; O'Donoghue et al., 1999; Archbold et al., 2000; Tobey et al., 2000). Improving communication skills, however, depends on several factors since there is a great variability on the communication outcomes after cochlear implantation. For example, several studies demonstrate speech perception and production after cochlear implantation improves with increased experience using the devices (Mondain et al., 1997; Allen et al., 1998; Peng et al., 2004; Uziel et al., 2007; Tobey et al., 2011). However, the principles behind the speech intelligibility improvement and increased variability following cochlear implantation are not well understood.

It has been proposed that the speech intelligibility is associated with the duration of sentences. Osberger and Levitt (1979) suggested that a reduced speaking rate increases the speech intelligibility since errors such as excessive prolongation of speech segments, insertion of long pauses, introduction of adventitious sound, failure to temporally differentiate stressed and unstressed syllables, and failure to modify segment duration as a function of the phonetic environment, are less likely to occur. It is not completely understood, however, how the duration of sentences is associated with speech intelligibility in children with cochlear implants. Another factor, which influences speech intelligibility is the role of context (McGarr, 1981). Judges award higher speech intelligibility scores when they listen to more predictable versus less predictable sentences. The purpose of this study is to further explore how increased experience with the cochlear implant influences sentence duration and speech intelligibility when context and syllabic length of the sentences are varied.

\section{METHODS}

\section{Participants}

107 children implanted before age of 4 years participated in the study. All participants were from large cohort of the children composed from the first cochlear implant recipients in North America. They were tested: first, when they were between 8 and 9 years old and second, when they were between 15 and 16 years of age. Children were residents of 33 states and 5 Canadian provinces (Geers and Brenner, 2003; Geers, 2006; Geers et al., 2011).

\section{Materials}

The 36 McGarr sentences (McGarr, 1983) were composed of 3, 5 and 7 syllables. Each sentence contained a monosyllabic key word: half of the sentences contained high predictability key words (high context) and half contained low predictability (low context) key words (Smith, 1975).

\section{Speech Intelligibility Testing}

Children repeated the sentences and their sentences were recorded on a DAT recorder with a microphone placed 12 inches from the child. Three normal hearing listeners heard, independently, one sentence from one child and wrote down the words they understood (an average of 108 listeners per child) and the average score was calculated. Speech intelligibility was defined as the number of correct words over the total words in the sentences.

\section{Duration of Sentences}


The sentences were analyzed using acoustic software to define the beginning and end of each sentence. The files were displayed as waveforms and duration of sentences was measured between the beginning and the end of each sentence. Total duration of sentences was computed and then the average duration across the 36 sentences was calculated in msec.

\section{RESULTS}

\section{Relationship between Duration of Sentences and Intelligibility Scores}

Figures 1 displays the relationships between duration of sentences and intelligibility scores for the two test ages of 8 and 16 years. Both correlation analyses revealed a significant negative correlation between children's intelligibility scores and duration of sentences at 8 years of age and 16 years of age [for 8 years: $r(105)=-0.454, p$ $<0.001$; for 16 years: $r(105)=-0.501, p<0.001]$. These data reinforce previous studies (Monsen, 1978; Osberger and Levitt, 1979; Tobey et al., 2003; Uchanski and Geers, 2003; Tobey et al., 2011) which showed longer sentence durations were associated with poorer intelligibility.

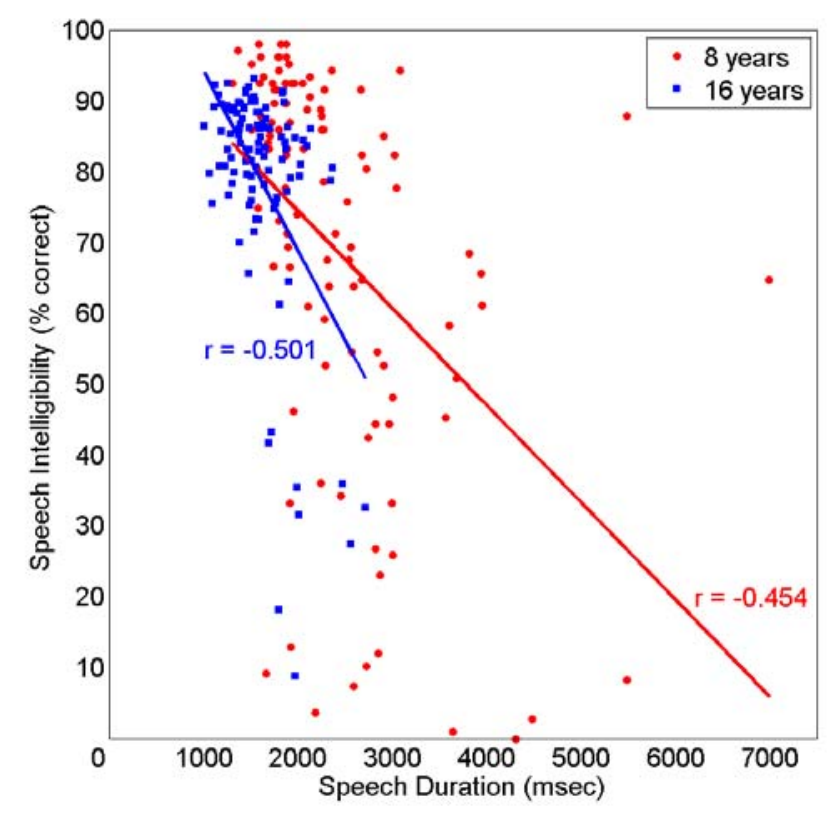

FIGURE 1. Scatter plot $(n=107)$ between duration of sentences and speech intelligibility. Each point indicates data from one listener. Green dots indicate 16 years and red dots indicate 8 years.

\section{Influence of Cochlear Implant Experience on Speech Intelligibility and Duration of Sentences}

Figure 2a indicates mean speech intelligibility and speech duration as a function of device experience, context, and number of syllables. In order to evaluate the influence of CI experience on speech intelligibility, a 3way repeated measures ANOVA with follow-up analyses using LSD procedure $(\mathrm{p}=0.05)$ was performed between age (8 versus 16 years), context information (high versus low), and number of syllables $(3,5,7)$ as they relate to speech intelligibility scores. There was a significant main effect of device experience $[F(1,106)=34.871, p<$ $0.001]$, a significant main effect of context $[F(1,106)=144.434, p<0.001]$, and a significant main effect for number of syllables $[F(2,212)=27.508, p<0.001]$. There was a significant 3 -way interaction $[F(2,212)=5.146$, $p=0.007$, Mse $=97.564]$. Examination of the cell means using $\left(\operatorname{LSD}_{\mathrm{mmd}}=2.673\right)$ indicated that at 8 years of age there was no significant difference in speech intelligibility between 3 and 5 syllable high context sentences and 3 and 7 syllable high context sentences. However, speech intelligibility of 5 syllable high context sentences was significantly higher than 7 syllable sentences. For low context sentences, there was no significant difference in intelligibility between 3 and 5 syllable sentences and both were significantly higher than the speech intelligibility of 
7 syllable low context sentences. However, at 16 years of age, there was no significant difference in intelligibility between 3 and 5 syllable high context sentences and both were significantly higher than the speech intelligibility of 7 syllable high context sentences. For low context sentences, there was no significant difference in intelligibility between 5 and 7 syllable sentences and both were significantly lower than the speech intelligibility of 3 syllable low context sentences.

In order to evaluate the influence of cochlear implant experience on duration of speech, a 3-way repeated measures ANOVA with follow-up analyses using LSD procedure $(\mathrm{p}=0.05)$ was performed between age, context information, and number of syllables. There was a significant main effect of device experience $[F(1,106)=98.623$, $p<0.001]$ and significant main effect for number of syllables $[F(2,212)=461.145, p<0.001]$. However, there was no significant effect of context on speech duration. There was a significant 3 -way interaction $[F(2,212)=6.223, p$ $=0.002]$ as shown in Figure $2 \mathrm{~b}$. Examination of the cell means using $\left(\mathrm{LSD}_{\mathrm{mmd}}=69.55\right)$ indicated that as the number of syllables increased, the total duration of the sentence increased and the increase in durations was higher for 8 years as compared to 16 years.
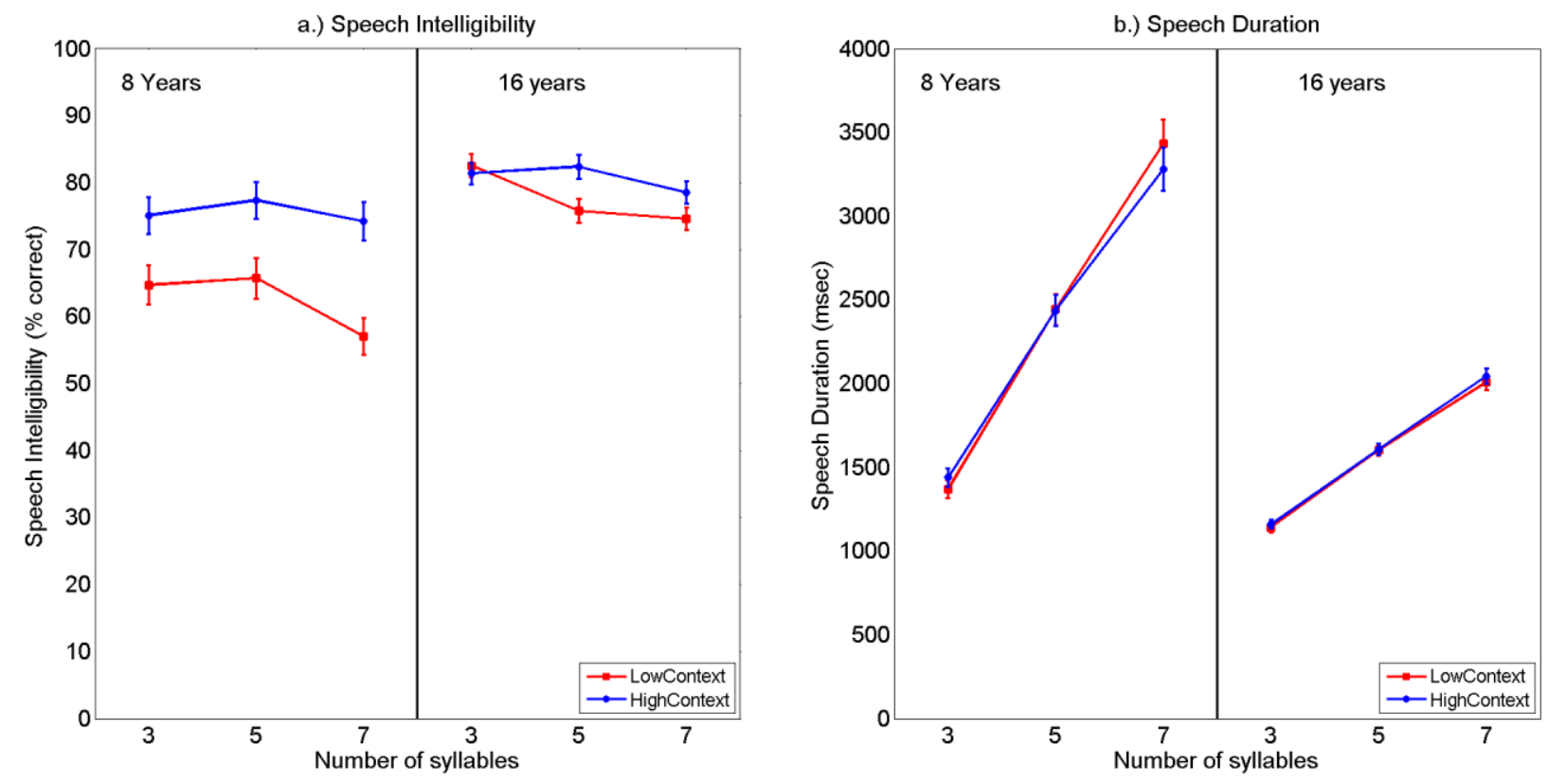

FIGURE 2. Mean speech intelligibility and speech duration as a function of device experience, context, and number of syllables. Panel a) indicates speech intelligibility and panel b) indicates speech duration.

\section{Influence of High and Low Context on Speech Intelligibility.}

Figure 3 indicates mean speech intelligibility for low and high context sentences for 8 years and 16 years. A within-groups factorial ANOVA with follow-up analyses using the LSD procedure $(p=0.05)$ was performed to evaluate the effect of context and age on speech intelligibility. There was an interaction between age and context as they relate to speech intelligibility $[F(1,106)=78.274, p<0.001]$. As hypothesized, the pattern of this interaction was that while speech intelligibility was consistently higher for high context sentences than the low context sentences, this effect was smaller at 16 years of age as compared to 8 years age $\left(\mathrm{LSD}_{\mathrm{mmd}}=1.595\right)$. 


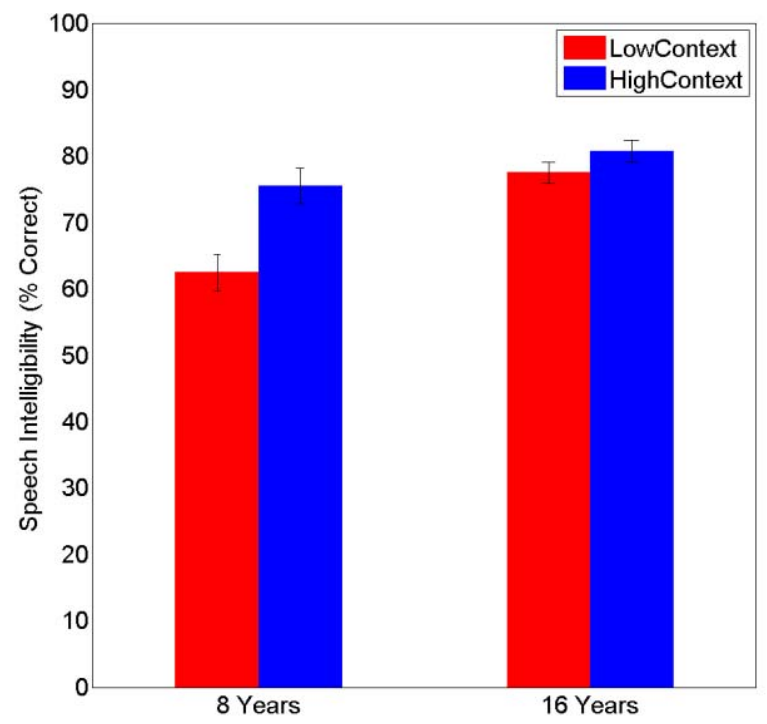

FIGURE 3. Effect of context on speech intelligibility for ages 8 and 16.

\section{DISCUSSION}

Data from this study concurs with data previously published by several authors (Monsen, 1978; Osberger and Levitt, 1979; McGarr, 1981; 1983; Tobey et al., 1991; Robbins et al., 1995; Miyamoto et al., 1996; Mondain et al., 1997; Allen et al., 1998; Vieu et al., 1998; O'Donoghue et al., 1999; Archbold et al., 2000; Tobey et al., 2000; Tobey et al., 2003; Uchanski and Geers, 2003; Peng et al., 2004; Uziel et al., 2007; Tobey et al., 2011). Speech intelligibility is higher for older children than younger children who produce sentences with durations that approximate those of typical hearing peers. Speech intelligibility for sentences containing high context words is higher for children with cochlear implants than low context sentences but the difference between high and low context sentences was greatest in the younger children. Context plays no role in duration measures.

The relationship between speech intelligibility and sentence duration indicates that the longer it takes to pronounce a sentence, the less intelligible sentence is. This observation is consistent with the findings of others (Monsen, 1978; Osberger and Levitt, 1979) who showed that children with HL demonstrate phonetic errors in their speech that makes it less intelligible. Monsen and Osberger reasoned that the speech intelligibility is reduce because of the decreased speaking rate, long pauses and additional sounds between phonemes and syllables. As the children acquire 8 years of experience with their devices from the first to second test session, sentence durations become shorter and speech intelligibility improves. We also found that the contextual information plays a significant role in the better understanding of the speech, as sentences that contain information that helps the listener to guess the meaning of the sentence results in higher intelligibility.

It is noteworthy to highlight two results: 1) we did not observe any effect of the contextual information on the duration of the sentence and 2) we did not observe any effect of the number of syllables over intelligibility when the number of syllables was lower than 5. Since all the contextual keywords were monosyllabic, it is not surprising that no contextual influence was observed. Duration of sentences between 3 and 5 syllables of length is not significantly different, and as a result, their speech intelligibilities are not significantly different either.

\section{ACKNOWLEDGMENTS}

We thank NIH-NIDCD (R01 DC008335) for funding this work and the assistance of Ann E. Geers and Christine A. Brenner in data collection. 


\section{REFERENCES}

Allen, M. C., Nikolopoulos, T. P., and O'Donoghue, G. M. (1998). "Speech intelligibility in children after cochlear implantation," The American journal of otology 19, 742-746.

Archbold, S. M., Nikolopoulos, T. P., Tait, M., O'Donoghue, G. M., Lutman, M. E., and Gregory, S. (2000). "Approach to communication, speech perception and intelligibility after paediatric cochlear implantation," British journal of audiology 34, 257-264.

Geers, A., and Brenner, C. (2003). "Background and educational characteristics of prelingually deaf children implanted by five years of age," Ear and hearing $\mathbf{2 4}, 2 \mathrm{~S}-14 \mathrm{~S}$.

Geers, A. E. (2006). "Factors influencing spoken language outcomes in children following early cochlear implantation," Advances in oto-rhino-laryngology 64, 50-65.

Geers, A. E., Brenner, C. A., and Tobey, E. A. (2011). "Long-term outcomes of cochlear implantation in early childhood: sample characteristics and data collection methods," Ear and hearing 32, 2S-12S.

Gold, T. (1980). "Speech production in hearing-impaired children," Journal of communication disorders 13, $397-418$.

McGarr, N. S. (1981). "The effect of context on the intelligibility of hearing and deaf children's speech," Language and speech 24, 255-264.

McGarr, N. S. (1983). "The intelligibility of deaf speech to experienced and inexperienced listeners," Journal of speech and hearing research 26, 451-458.

Miyamoto, R. T., Kirk, K. I., Robbins, A. M., Todd, S., and Riley, A. (1996). "Speech perception and speech production skills of children with multichannel cochlear implants," Acta oto-laryngologica 116, 240-243.

Mondain, M., Sillon, M., Vieu, A., Lanvin, M., Reuillard-Artieres, F., Tobey, E., and Uziel, A. (1997). "Speech perception skills and speech production intelligibility in French children with prelingual deafness and cochlear implants," Archives of otolaryngology--head \& neck surgery 123, 181-184.

Monsen, R. B. (1978). "Toward measuring how well hearing-impaired children speak," Journal of speech and hearing research 21, 197-219.

O'Donoghue, G. M., Nikolopoulos, T. P., Archbold, S. M., and Tait, M. (1999). "Cochlear implants in young children: the relationship between speech perception and speech intelligibility," Ear and hearing 20, 419-425.

Osberger, M. J., and Levitt, H. (1979). "The effect of timing errors on the intelligibility of deaf children's speech," The Journal of the Acoustical Society of America 66, 1316-1324.

Peng, S. C., Spencer, L. J., and Tomblin, J. B. (2004). "Speech intelligibility of pediatric cochlear implant recipients with 7 years of device experience," Journal of speech, language, and hearing research : JSLHR 47, 1227-1236.

Robbins, A. M., Kirk, K. I., Osberger, M. J., and Ertmer, D. (1995). "Speech intelligibility of implanted children," The Annals of otology, rhinology \& laryngology. Supplement 166, 399-401.

Smith, C. R. (1975). "Residual hearing and speech production in deaf children," Journal of speech and hearing research 18, 795811.

Tobey, E. A., Angelette, S., Murchison, C., Nicosia, J., Sprague, S., Staller, S. J., Brimacombe, J. A., and Beiter, A. L. (1991). "Speech production performance in children with multichannel cochlear implants," The American journal of otology 12 Suppl, 165-173.

Tobey, E. A., Geers, A. E., Brenner, C., Altuna, D., and Gabbert, G. (2003). "Factors associated with development of speech production skills in children implanted by age five," Ear and hearing 24, 36S-45S.

Tobey, E. A., Geers, A. E., Douek, B. M., Perrin, J., Skellet, R., Brenner, C., and Toretta, G. (2000). "Factors associated with speech intelligibility in children with cochlear implants," The Annals of otology, rhinology \& laryngology. Supplement 185, 28-30.

Tobey, E. A., Geers, A. E., Sundarrajan, M., and Shin, S. (2011). "Factors influencing speech production in elementary and high school-aged cochlear implant users," Ear and hearing 32, 27S-38S.

Uchanski, R. M., and Geers, A. E. (2003). "Acoustic characteristics of the speech of young cochlear implant users: a comparison with normal-hearing age-mates," Ear and hearing 24, 90S-105S.

Uziel, A. S., Sillon, M., Vieu, A., Artieres, F., Piron, J. P., Daures, J. P., and Mondain, M. (2007). "Ten-year follow-up of a consecutive series of children with multichannel cochlear implants," Otology \& neurotology : official publication of the American Otological Society, American Neurotology Society [and] European Academy of Otology and Neurotology 28, 615-628.

Vieu, A., Mondain, M., Blanchard, K., Sillon, M., Reuillard-Artieres, F., Tobey, E., Uziel, A., and Piron, J. P. (1998). "Influence of communication mode on speech intelligibility and syntactic structure of sentences in profoundly hearing impaired French children implanted between 5 and 9 years of age," International journal of pediatric otorhinolaryngology 44, 1522. 